\title{
Factors Associated with Refusal of Rapid HIV Testing in an Emergency Department
}

\author{
Mary L. Pisculli • William M. Reichmann • Elena Losina • \\ Laurel A. Donnell-Fink • Christian Arbelaez • Jeffrey N. Katz • \\ Rochelle P. Walensky
}

Published online: 27 October 2010

(C) The Author(s) 2010. This article is published with open access at Springerlink.com

\begin{abstract}
HIV screening studies in the emergency department (ED) have demonstrated rates of HIV test refusal ranging from $40-67 \%$. This study aimed to determine the factors associated with refusal to undergo routine rapid HIV testing in an academic ED in Boston. HIV counselors offered routine testing to 1,959 patients; almost one-third of patients $(29 \%)$ refused. Data from a selfadministered survey were used to determine independent correlates of HIV testing refusal. In multivariate analysis, women and patients with annual household incomes of $\$ 50,000$ or more were more likely to refuse testing, as were those who reported not engaging in HIV risk behaviors, those previously HIV tested and those who did not perceive a need for testing. Enrollment during morning hours was also associated with an increased risk of refusal. Increased educational efforts to convey the rationale and benefits of universal screening may improve testing uptake among these groups.
\end{abstract}

M. L. Pisculli · R. P. Walensky

Division of Infectious Disease, Brigham and Women's Hospital, Boston, MA, USA

W. M. Reichmann - E. Losina - L. A. Donnell-Fink - J. N. Katz Department of Orthopedic Surgery, Brigham and Women's Hospital, Boston, MA, USA

W. M. Reichmann · E. Losina

Department of Biostatistics, Boston University School of Public Health, Boston, MA, USA

C. Arbelaez

Department of Emergency Medicine, Brigham and Women's Hospital, Boston, MA, USA

J. N. Katz

Division of Rheumatology, Immunology and Allergy, Brigham and Women's Hospital, Boston, MA, USA
Keywords Rapid HIV testing - HIV test refusal · HIV test acceptance · Routine HIV testing · Emergency department $\cdot$ Human immunodeficiency virus

\section{Introduction}

The Centers for Disease Control and Prevention (CDC) estimate that 1 in 5 persons living with HIV infection in the United States is unaware of his or her diagnosis, accounting for more than 232,000 undiagnosed cases of HIV infection [1]. To facilitate timely detection, the CDC revised their HIV testing guidelines in 2006 to recommend routine HIV testing in all health care settings, including emergency departments (ED), for patients aged 13-64 years [2]. Despite these recommendations, in a survey of academic

\author{
J. N. Katz \\ Department of Epidemiology, Harvard School of Public Health, \\ Boston, MA, USA \\ R. P. Walensky \\ Division of Infectious Disease, Massachusetts General Hospital, \\ Boston, MA, USA \\ R. P. Walensky \\ Center for AIDS Research, Harvard Medical School, Boston, \\ MA, USA \\ R. P. Walensky $(\varangle)$ \\ Division of Infectious Disease, Massachusetts General Hospital, \\ 50 Staniford Street, 9th Floor, Boston, MA 02114, USA \\ e-mail: rwalensky@ partners.org
}


EDs conducted in December 2006 through March 2007, only $13 \%$ offered routine HIV screening [3]. As such, HIV testing remains underutilized, with only $37 \%$ of US adults having ever been tested for HIV [4].

As HIV infection is no longer confined to traditional high-risk groups [1], screening for HIV infection in ED settings has the potential to extend testing to a broader population, including heterosexual men and women, racial and ethnic minorities, and socio-economically disadvantaged persons who may be more likely to seek care in an ED setting [5-7]. EDs provide health care to persons at risk of HIV infection in part due to either a lack of insurance or a primary care physician. Many newly diagnosed HIVinfected patients have had multiple encounters in ED settings prior to diagnosis, representing "missed opportunities" for earlier detection [8, 9]. Previous studies of routine voluntary HIV testing interventions in ED and urgent care settings have been limited by test refusal rates as high as 40-67\% [10-13]. While prior studies collected basic demographic information and patients' self-reported reason for refusal [11-13], they did not, however, match reasons for refusal in ED settings with patients' reported HIV risk factors to determine if risk perception and associated refusal were appropriate.

A more thorough understanding of the factors underlying HIV test refusal is critical as test refusers may in fact be at a higher risk of HIV infection than those who accept [14, 15]. In this report, we aim to identify correlates of refusal to undergo HIV testing in the ED. We hypothesized that the patient-specific reasons for refusing testing are likely multifactorial and potentially include access to an alternative regular source of care (and presumably to testing), limited HIV-related knowledge, and a low perception of risk and/or negative attitudes toward routine testing.

\section{Methods}

\section{Parent Study}

This study was conducted within the Universal Screening for HIV-infection in the Emergency Room (USHER) trial, funded by the National Institutes of Health; details of the USHER trial have been published elsewhere [16-18]. The parent study was conducted in the ED of Brigham and Women's Hospital, a tertiary academic medical center in Boston, Massachusetts. This ED serves over 56,000 patients annually, $48 \%$ of whom are White, $25 \%$ are Black and $20 \%$ Hispanic. Approximately $40 \%$ are men; the median age is 44 years.

Patients were eligible to enroll in the USHER trial if they were awaiting care in the ED when an HIV counselor was available and were between the ages of 18-75 years,
English- or Spanish-speaking, and not known to be HIV infected. Patients receiving prenatal care and those with an altered mental status or an emergency severity index (ESI) score of 1 or 2 (used as a surrogate for poor mental status or increased acuity) were excluded from study participation. The five-tiered ESI algorithm is employed at ED triage to assess both patient acuity and the anticipated utilization of ED resources - a score of 1 reflects the highest resourceintensity and 5 the least [19]. Enrollment periods varied weekly and spanned the hours between 8 am and 12 am, Sunday through Saturday, for a minimum of 60 enrollment hours per week.

Eligible patients were approached by a bilingual (English-Spanish) HIV counselor and invited to enroll in a trial designed to learn more about HIV testing. Upon consent to the trial, subjects were asked to complete a 20-min, 86-item self-administered questionnaire. Subjects elected to complete the questionnaire as either a pencil-and-paper version or an audio computer-assisted self-interview (ACASI). Both questionnaire modalities were administered in the patient's private room within the ED. Neither the HIV counselors nor other ED study staff had access to the results with the rare exception when a participant requested assistance completing the questionnaire.

Enrolled subjects were randomized to rapid HIV test offer by either an emergency provider or a dedicated HIV counselor. During the 17-month trial period, 28 emergency service (nursing) assistants (provider arm) and 9 USHERdedicated HIV counselors (counselor arm) were trained to consent and test participants. Due to a low test offer rate in the provider arm (36\%) compared to the counselor arm $(80 \%)$ [18], we elected to examine correlates of refusal only from the counselor arm to minimize concerns of possible targeted test offer-with associated motivations to accept - in the provider arm, based on HIV-related symptoms. Trial participants were offered a rapid oral HIV antibody test (OraQuick ${ }^{\circledR}$ Advance Rapid HIV-1/2 Antibody Test, OraSure Technologies, Bethlehem, Pennsylvania) free of charge with confirmation of reactive results as previously described. Subjects who consented to rapid HIV testing provided separate written informed consent for rapid HIV testing in accordance with Massachusetts state law. Trial participants were not offered any financial incentives. The USHER trial was approved by the Partners Human Research Committee (protocol 2006P-000136) and overseen by a Data Safety Monitoring Board.

Data Collection Instrument

\section{Demographics and Access to Care}

Demographic data (gender, age, race/ethnicity, primary language and education level) were obtained at the time of 
enrollment by the HIV counselor. The subjects' chief complaint and diagnosis were not recorded in trial-related documents. The questionnaire collected self-reported information on annual household income, medical insurance status, access to a primary care provider, risk factor assessment of substance abuse and sexual practices, HIVrelated knowledge, self-perceived need for HIV testing, and HIV testing history. Annual household income was reported as less than $\$ 20,000, \$ 20-50,000, \$ 50-100,000$ and greater than $\$ 100,000$ and, based on response distribution, was categorized as less than $\$ 50,000$ versus $\$ 50,000$ or more. Medical insurance status was obtained by asking participants to indicate all sources of coverage from the following options: Medicare, Medicaid, private and/or other ("insured") or uninsured. Participants were also asked if they had a primary care provider ("yes" versus "no" or "I don't know").

\section{HIV Risk Behaviors, HIV Knowledge, Perceived Need for Testing and History of Prior Testing of Refusers}

To examine the influence of HIV risk behaviors, participants were asked a series of questions regarding sexual behaviors and frequency of illicit drug behavior or alcohol use. We defined sexual risk as report of any of the following: history of a sexually transmitted infection; two or more sexual partners in the past year; any history of men having sex with men; inconsistent or no condom use; history of incarceration; or sex with a partner who was known to have been incarcerated, HIV infected or who used recreational drugs.

Illicit drug use was defined as report of any of the following: any history of injection drug use or a response of "occasionally" or more frequent use of marijuana, cocaine/ crack, amphetamines, crystal methamphetamine, alkyl nitrites, LSD, ecstasy, heroin and/or oxycontin, or other narcotics. Patients were asked to characterize their frequency of use of these illicit substances as "never," "once," "occasionally," "monthly," or "more than once per month". Answering "once" to two or more drugs was also considered illicit drug behavior.

Alcohol use was included in the assessment of HIV risk behaviors because previous studies have demonstrated a relationship between alcohol use disorders and sexual risk behaviors [20, 21]. Alcohol risk behavior was defined according to the 10-question Alcohol Use Disorders Identification Test (AUDIT) survey developed by the World Health Organization; an AUDIT score of 8 or greater is consistent with "hazardous and harmful" alcohol use [22].

Participants who reported at least one of these three risk behaviors (sexual, illicit drug, alcohol) were categorized as having an HIV risk behavior. Those who reported not engaging in all queried risk factors were categorized as not having any risk factors. Participants who reported no risk factors but did not provide complete information for all risk behaviors were categorized as 'missing'.

Knowledge of HIV transmission and self-protective behaviors was measured using the 18-item validated HIV Knowledge Scale (HIV-KQ-18) [23]. HIV-related knowledge scores were treated as a dichotomous variable, "high" ( $\geq 17$ correct items) versus "low" ( $\leq 16$ correct), based on an a priori hypothesis that those with imperfect or "low" knowledge may be more likely to refuse HIV testing. Sensitivity analyses were undertaken considering the HIV knowledge score as a continuous variable. HIV testing history was obtained by the following question: "Have you ever been tested for HIV, the virus that causes AIDS?" Self-perceived need for HIV screening was defined as agreement ("strongly agree" or "agree" versus "undecided", "disagree" or "strongly disagree") with the statement, "Based on my risk for HIV infection, I think I should be HIV tested". We also examined whether enrollment day (weekend vs. weekday) and time ( 8 am to $2 \mathrm{pm}, 2 \mathrm{pm}$ to $6 \mathrm{pm}, 6 \mathrm{pm}$ to 12am) were associated with refusal rates.

\section{Statistical Analysis}

The outcome of interest was HIV testing refusal. Refusal rate was defined as the proportion of study participants who refused to undergo testing among those who were enrolled in the trial and offered HIV screening by an HIV counselor. As refusal was relatively common, we used modified Poisson regression with robust variance estimation to estimate the relative risk (RR) as a measure of association for each risk factor under consideration and risk of refusal [24].

A multivariate Poisson regression model was built to identify independent correlates of higher refusal rates. Inclusion of each covariate was based on either evidence from prior studies, clinical judgment, author consensus or having reached a provisional threshold for statistical significance in bivariate analysis $(p<0.1)$. Factors included in the multivariate model were gender, age, race/ethnicity, education, annual household income, report of HIV risk behaviors, level of HIV knowledge, history of prior testing, perceived need for testing and time and day of enrollment. Due to a high degree of collinearity with race/ethnicity, language was excluded from the final model. Items to which study participants did not provide answers were marked as a separate category noted as 'missing' and interpreted as "lack of disclosure". In secondary analyses we characterize correlates of refusal among participants who perceived a need for HIV screening. Covariates that reached a threshold $p$ value of $<0.1$ on bivariate analysis were advanced to a multivariate Poisson regression model. All analyses were conducted using SAS software version 9.1.3 (SAS Institute, Cary, NC). 


\section{Results}

\section{Study Sample}

Between February 2007 and June 2008, 12,970 ED patients were screened for USHER trial eligibility based on age, English/Spanish speaking and ESI score. Of the 8,187 eligible patients approached, 4,860 (59\%) agreed to participate in the USHER trial and 4,855 were randomized to either the counselor $(2,446)$ or provider $(2,409)$ arms [18]. Eligible patients who refused trial enrollment were similar in gender and ESI score distribution to trial participants; trial enrollees were significantly younger than those who refused participation (37 vs. 43 years, $p<0.0001$ ).

Among the 1,959 subjects offered HIV testing by an HIV counselor, 577 (29\%) refused. Demographic characteristics of these subjects are presented in Table 1 . Women represented $65 \%$ of our study sample. The median age was 35 years (interquartile range, 25-48 years) and 38\% were White, $21 \%$ were Black and $30 \%$ were Hispanic. About

Table 1 Demographics of subjects offered a rapid HIV test by an HIV counselor, $N=1959$

\begin{tabular}{|c|c|}
\hline Characteristic & Number $\left(\%^{\mathrm{a}}\right)$ \\
\hline Total & 1959 \\
\hline \multicolumn{2}{|c|}{ Gender, $n=1951$} \\
\hline Men & $679(34.8 \%)$ \\
\hline Women & $1272(65.2 \%)$ \\
\hline Age, $n=1919$ & Median $35\left[\mathrm{IQR}^{\mathrm{b}}, 25-48\right.$ \\
\hline $18-29$ & $731(38.1 \%)$ \\
\hline $30-39$ & $404(21.1 \%)$ \\
\hline $40-49$ & $359(18.7 \%)$ \\
\hline $50-59$ & $260(13.6 \%)$ \\
\hline $60+$ & $165(8.6 \%)$ \\
\hline \multicolumn{2}{|c|}{ Race/ethnicity, $n=1944$} \\
\hline White & $744(38.3 \%)$ \\
\hline Black & $417(21.4 \%)$ \\
\hline Hispanic & $585(30.1 \%)$ \\
\hline Other ${ }^{\mathrm{c}}$ & $198(10.2 \%)$ \\
\hline \multicolumn{2}{|c|}{ Primary language, $n=1946$} \\
\hline English & $1415(72.7 \%)$ \\
\hline Spanish & $409(21.0 \%)$ \\
\hline Other & $122(6.3 \%)$ \\
\hline \multicolumn{2}{|c|}{ Education, $n=1951$} \\
\hline$<$ High school & $259(13.3 \%)$ \\
\hline High school & $446(22.9 \%)$ \\
\hline$>$ High school & $1246(63.9 \%)$ \\
\hline
\end{tabular}

${ }^{\text {a }}$ Percentages may total more than $100 \%$ due to rounding

b IQR-Interquartile range

c Due to small numbers, participants who identified themselves as either Asian, Native-American, multiracial or other were all categorized as Other one-quarter $(27 \%)$ of participants did not speak English as their primary language. Nearly two-thirds (64\%) of participants reported their educational attainment to be past high school.

\section{Demographic Characteristics and Access to Care} of Test Refusers

Bivariate analyses (Table 2) demonstrated that women were more likely to refuse testing than men, as were persons 30 years or older. Hispanic subjects and Spanish speakers were less likely to refuse testing than Whites and English speakers, respectively. Both educational attainment greater than high school and an annual household income of more than $\$ 50,000$ were associated with increased refusal. We did not find an association between HIV test refusal and access to care as defined by insurance status or having a primary care provider.

HIV Risk Behaviors, HIV Knowledge, History of Prior Testing and Perceived Need for Testing of Refusers

Participants who did not report or disclose HIV risk behaviors were more likely to refuse testing than those who reported any HIV risk behavior. Nearly two-thirds of refusers (367 of 577) had low HIV-related knowledge scores; however, we did not find a statistically significant or clinically meaningful association between HIV test refusal and HIV knowledge in bivariate analysis. Results of sensitivity analyses considering HIV knowledge score as a continuous variable did not change our conclusions.

Of participants who perceived a need for testing, $14.9 \%$ refused testing. Those participants who did not perceive a need for testing as well as those who did not provide a response regarding perception of need for testing were more likely to refuse. Of note, among all subjects offered testing who reported an HIV risk behavior, only $37.9 \%$ perceived a need for testing. Among subjects who refused testing and reported an HIV risk behavior, even fewer, $15.6 \%$, perceived a need for testing.

Time of Enrollment

Participants enrolled in the morning hours ( 8 am-2 pm) were more likely to refuse than evening (6 pm-12 am) enrollees. A statistically significant association between HIV test refusal and day of enrollment (weekend vs. weekday) was not detected in this sample.

Results of Multivariate Analyses

In multivariate analysis, women $(\mathrm{RR}=1.23,95 \% \mathrm{CI}$ : 1.06-1.43) and participants with annual household incomes 
Table 2 Bivariate and multivariate analysis of correlates of refusal of rapid HIV screening

\begin{tabular}{|c|c|c|c|c|c|}
\hline \multirow[t]{2}{*}{ Characteristic } & \multirow{2}{*}{$\begin{array}{l}\text { Refused rapid HIV } \\
\text { test, } n=577\end{array}$} & \multicolumn{2}{|l|}{ Bivariate } & \multicolumn{2}{|c|}{ Multivariate } \\
\hline & & $\mathrm{RR}^{\mathrm{a}}$ & $95 \% \mathrm{CI}^{\mathrm{b}}$ & $\mathrm{RR}^{\mathrm{a}}$ & $95 \% \mathrm{CI}^{\mathrm{b}}$ \\
\hline \multicolumn{6}{|l|}{ Gender $^{\mathrm{c}}$} \\
\hline Men & $177(26.1 \%)$ & Reference & & & \\
\hline Women & $398(31.3 \%)$ & 1.20 & $1.03-1.40$ & 1.23 & $1.06-1.43$ \\
\hline \multicolumn{6}{|l|}{$\mathrm{Age}^{\mathrm{c}}$} \\
\hline $18-29$ & $183(25.0 \%)$ & Reference & & & \\
\hline $30+$ & $381(32.1 \%)$ & 1.28 & $1.10-1.49$ & 1.11 & $0.95-1.29$ \\
\hline \multicolumn{6}{|l|}{ Race/ethnicity ${ }^{c}$} \\
\hline White & $256(34.4 \%)$ & Reference & & & \\
\hline Black & $127(30.5 \%)$ & 0.89 & $0.74-1.06$ & 1.04 & $0.87-1.24$ \\
\hline Hispanic & $135(23.1 \%)$ & 0.67 & $0.56-0.80$ & 0.86 & $0.70-1.05$ \\
\hline Other & $57(28.8 \%)$ & 0.84 & $0.66-1.06$ & 0.89 & $0.70-1.13$ \\
\hline \multicolumn{6}{|l|}{ Primary language $\mathrm{c}^{\mathrm{c}}$} \\
\hline English & $449(31.7 \%)$ & Reference & & & \\
\hline Spanish & $94(23.0 \%)$ & 0.72 & 0. $60-0.88$ & - & - \\
\hline Other & $31(25.4 \%)$ & 0.80 & $0.59-1.10$ & - & - \\
\hline \multicolumn{6}{|l|}{ Education $^{\mathrm{c}}$} \\
\hline$<$ High school & $61(23.6 \%)$ & Reference & & & \\
\hline High school & $117(26.2 \%)$ & 1.11 & $0.85-1.46$ & 1.09 & $0.82-1.43$ \\
\hline$>$ High school & $395(31.7 \%)$ & 1.35 & $1.07-1.70$ & 1.09 & $0.84-1.40$ \\
\hline \multicolumn{6}{|c|}{ Annual household income } \\
\hline$<\$ 50,000$ & $191(23.9 \%)$ & Reference & & & \\
\hline$\geq \$ 50,000$ & $179(36.5 \%)$ & 1.53 & $1.29-1.81$ & 1.25 & $1.04-1.51$ \\
\hline Missing & $207(30.9 \%)$ & 1.29 & $1.09-1.53$ & 1.13 & $0.86-1.47$ \\
\hline \multicolumn{6}{|l|}{ Insurance status } \\
\hline Insured & $386(29.2 \%)$ & Reference & & & \\
\hline Uninsured & $16(24.6 \%)$ & 0.84 & $0.55-1.30$ & - & - \\
\hline Missing & $175(30.6 \%)$ & 1.05 & $0.90-1.22$ & - & - \\
\hline \multicolumn{6}{|c|}{ Has a primary care provider } \\
\hline Yes & $313(28.4 \%)$ & Reference & & & \\
\hline No/don’t know & $70(30.7 \%)$ & 1.08 & $0.87-1.34$ & - & - \\
\hline Missing & $194(30.9 \%)$ & 1.09 & $0.94-1.27$ & - & - \\
\hline \multicolumn{6}{|l|}{ HIV risk behavior } \\
\hline Present & $289(25.3 \%)$ & Reference & & & \\
\hline None reported & $66(39.5 \%)$ & 1.56 & $1.26-1.93$ & 1.43 & $1.15-1.78$ \\
\hline Missing & $222(34.1 \%)$ & 1.35 & $1.16-1.56$ & 1.59 & $1.23-2.06$ \\
\hline \multicolumn{6}{|l|}{ HIV knowledge } \\
\hline High & $97(29.6 \%)$ & Reference & & & \\
\hline Low & $367(30.5 \%)$ & 1.03 & $0.85-1.24$ & 1.14 & $0.94-1.38$ \\
\hline Missing & $113(26.5 \%)$ & 0.90 & $0.71-1.13$ & 0.74 & $0.52-1.07$ \\
\hline \multicolumn{6}{|l|}{ Prior HIV test } \\
\hline No prior HIV test & $130(28.3 \%)$ & Reference & & & \\
\hline Prior HIV test & $251(28.0 \%)$ & 0.99 & $0.83-1.19$ & 1.20 & 1.01-1.44 \\
\hline Missing & $196(32.5 \%)$ & 1.15 & $0.95-1.38$ & 1.44 & $1.03-2.01$ \\
\hline \multicolumn{6}{|c|}{ Perceived need for testing } \\
\hline Perceived need & $93(14.9 \%)$ & Reference & & & \\
\hline No perceived need & $364(40.7 \%)$ & 2.74 & $2.24-3.36$ & 2.60 & $2.10-3.23$ \\
\hline Missing & $120(27.3 \%)$ & 1.84 & $1.44-2.34$ & 1.57 & $1.11-2.22$ \\
\hline
\end{tabular}


Table 2 continued

\begin{tabular}{|c|c|c|c|c|c|}
\hline \multirow[t]{2}{*}{ Characteristic } & \multirow{2}{*}{$\begin{array}{l}\text { Refused rapid HIV } \\
\text { test, } n=577\end{array}$} & \multicolumn{2}{|c|}{ Bivariate } & \multicolumn{2}{|c|}{ Multivariate } \\
\hline & & $\mathrm{RR}^{\mathrm{a}}$ & $95 \% \mathrm{CI}^{\mathrm{b}}$ & $\mathrm{RR}^{\mathrm{a}}$ & $95 \% \mathrm{CI}^{\mathrm{b}}$ \\
\hline \multicolumn{6}{|l|}{ Time of enrollment ${ }^{\mathrm{c}}$} \\
\hline Evening $6 \mathrm{pm}-12 \mathrm{am}$ & $119(25.7 \%)$ & Refe & & & \\
\hline Afternoon $2 \mathrm{pm}-6 \mathrm{pm}$ & $203(28.6 \%)$ & 1.11 & $0.92-1.35$ & 1.09 & $0.90-1.32$ \\
\hline Morning 8am-2 pm & $250(32.3 \%)$ & 1.26 & $1.04-1.51$ & 1.24 & $1.03-1.49$ \\
\hline \multicolumn{6}{|l|}{ Day of enrollment ${ }^{\mathrm{c}}$} \\
\hline Weekend & $89(25.7 \%)$ & Refe & & & \\
\hline Weekday & $486(30.2 \%)$ & 1.17 & $0.97-1.43$ & 1.17 & $0.97-1.41$ \\
\hline \multicolumn{6}{|c|}{${ }^{\text {a }}$ Relative risk $(\mathrm{RR})>1$ indicates increased refusal of rapid HIV testing versus the reference group } \\
\hline
\end{tabular}

of at least $\$ 50,000(\mathrm{RR}=1.25,95 \% \mathrm{CI}: 1.04-1.51)$ were more likely to refuse HIV testing (Table 2). Furthermore, participants who reported engaging in no HIV risk behavior had an increased risk of refusal $(\mathrm{RR}=1.43,95 \% \mathrm{CI}$ : 1.15-1.78), as did those who did not completely disclose their HIV risk behavior ( $\mathrm{RR}=1.59,95 \% \mathrm{CI}: 1.23-2.06)$. Compared to those without a prior HIV testing history, both those study participants who had been tested for HIV previously $(\mathrm{RR}=1.20,95 \% \mathrm{CI}: 1.01-1.44)$ and those who did not provide any information regarding prior HIV testing history $(\mathrm{RR}=1.44,95 \% \mathrm{CI}$ : $1.03-2.01)$ were more likely to refuse HIV testing. Patients who either did not perceive a need for testing $(\mathrm{RR}=2.60,95 \% \mathrm{CI}$ : 2.10-3.23) or whose response to a perceived need were missing $(\mathrm{RR}=1.57,95 \% \mathrm{CI}: 1.11-2.22)$ were also more likely to refuse. Morning hours were associated with higher rates of refusal $(\mathrm{RR}=1.24,95 \% \mathrm{CI}: 1.03-1.49)$.

Correlates of Refusal Among Those With a Perceived Need for Testing

Among the 1,959 subjects offered HIV testing by an HIV counselor, $626(32 \%)$ reported a perceived need for testing. Bivariate analyses of those who perceived a need for testing demonstrated the following significant correlates of refusal: age greater than 30 years; household income of greater than $\$ 50,000$; no HIV risk behaviors; low HIV knowledge; prior HIV testing; and weekday enrollment. Participants who did not report their insurance status were less likely to refuse. In multivariate analysis, only subjects who reported engaging in no HIV risk behaviors $(\mathrm{RR}=2.08$, 95\% CI: $1.08-3.97)$ and those who did not disclose their HIV risk behavior $(\mathrm{RR}=2.01,95 \% \mathrm{CI}$ : 1.03-3.93) were found to be more likely to refuse (Table 3).
Table 3 Multivariate analysis of correlates of refusal of rapid HIV screening among patients who perceived a need for HIV testing, $N=626$

\begin{tabular}{|c|c|c|c|}
\hline \multirow[t]{2}{*}{ Characteristic } & \multirow{2}{*}{$\begin{array}{l}\text { Refused rapid HIV } \\
\text { test, } n=93\end{array}$} & \multicolumn{2}{|c|}{ Multivariate } \\
\hline & & $\mathrm{RR}$ & $95 \% \mathrm{CI}$ \\
\hline \multicolumn{4}{|l|}{$\operatorname{Age}^{\mathrm{a}}$} \\
\hline $18-29$ & $29(11.4 \%)$ & \multicolumn{2}{|c|}{ Reference } \\
\hline $30+$ & $63(17.5 \%)$ & 1.23 & $0.81-1.88$ \\
\hline \multicolumn{4}{|c|}{ Annual household income } \\
\hline$<\$ 50,000$ & $40(11.2 \%)$ & \multicolumn{2}{|c|}{ Reference } \\
\hline$\geq \$ 50,000$ & $16(15.7 \%)$ & 1.46 & $0.83-2.55$ \\
\hline Missing & $37(22.2 \%)$ & 1.14 & $0.60-2.15$ \\
\hline \multicolumn{4}{|l|}{ Insurance status } \\
\hline Insured & $59(12.6 \%)$ & \multicolumn{2}{|c|}{ Reference } \\
\hline Uninsured & $4(9.8 \%)$ & 0.86 & $0.34-2.18$ \\
\hline Missing & $30(25.6 \%)$ & 0.90 & $0.45-1.80$ \\
\hline \multicolumn{4}{|l|}{ HIV risk behavior } \\
\hline Present & $45(10.4 \%)$ & \multicolumn{2}{|c|}{ Reference } \\
\hline None reported & $10(20.8 \%)$ & 2.08 & 1.08-3.97 \\
\hline Missing & $38(26.0 \%)$ & 2.01 & $1.03-3.93$ \\
\hline \multicolumn{4}{|l|}{ HIV knowledge } \\
\hline High & $14(14.6 \%)$ & \multicolumn{2}{|c|}{ Reference } \\
\hline Low & $64(13.3 \%)$ & 0.84 & $0.48-1.47$ \\
\hline Missing & $15(30.0 \%)$ & 1.27 & $0.60-2.71$ \\
\hline \multicolumn{4}{|l|}{ Prior HIV test } \\
\hline No prior HIV test & $10(7.5 \%)$ & \multicolumn{2}{|c|}{ Reference } \\
\hline Prior HIV test & $46(13.0 \%)$ & 1.77 & $0.92-3.42$ \\
\hline Missing & $37(27.0 \%)$ & 2.09 & $0.77-5.69$ \\
\hline \multicolumn{4}{|l|}{ Day of enrollment } \\
\hline Weekend & $11(9.4 \%)$ & \multicolumn{2}{|c|}{ Reference } \\
\hline Weekday & $82(16.1 \%)$ & 1.73 & $0.95-3.12$ \\
\hline
\end{tabular}

Bold values denote $p<0.05$

${ }^{\text {a }}$ Due to missing values, age, $n=92$ 


\section{Discussion}

Within the context of the USHER trial, 29\% of the 1,959 patients who were offered a rapid HIV test in the emergency department by an HIV counselor refused the test. This rate of refusal is comparable to the experience of other ED studies [10-13]. Despite the overall success of routine HIV testing programs, we identified women and participants with annual household incomes of $\$ 50,000$ or greater to be more likely to refuse testing, as well as those who reported no HIV risk behaviors, those previously tested for HIV, those who did not perceive a need for testing and participants enrolled during morning hours.

Complex factors including lack of social support and fear of stigma or rejection if HIV-infected may underlie the identified greater tendency of women to refuse testing [25]. Although women have the highest rate of lifetime HIV testing, much of this testing is performed in the context of prenatal care [26]. Women infected through heterosexual contact represent an increasing proportion of HIV infection in the U.S, underscoring the clinical relevance of our finding [27]. Low risk-perception has been postulated as a possible explanation for this rise in diagnosed infection among women [28] and this misperception may be shared by health care providers who may be less likely to offer HIV testing to women [9].

We also identified a significant association between higher incomes and increased HIV test refusal. This association has not been previously described; prior studies of routine ED HIV testing have not included income in their analysis [10, 29]. Higher income, however, has been correlated with having been previously tested in a study of rapid HIV testing among men who have sex with men frequenting bathhouses in New York [30]. Differences in the motivation for testing in a risk-taking venue versus an ED screening study may account for these observations.

Consistent with the results of National Health Interview Surveys, we also found that patients who reported not engaging in HIV risk behaviors were more likely to refuse than those who reported any HIV risk behavior [26]. Patients who did not disclose their HIV risk behaviors were also more likely to refuse. We do not have HIV seroprevalence data from this sample to determine if those who refused are indeed infected or have unreported HIV risk behaviors. In addition, we did not observe a significant association of test refusal with a low score on the HIV knowledge scale to suggest that a poor understanding of HIV transmission motivates test refusal.

After adjusting for other factors, we found a history of prior HIV testing to be associated with a $20 \%$ increase in HIV test refusal. Refusal for these participants may simply be related to close temporal proximity to their most recent test [2]; however, we are unable to corroborate dates of self-reported prior testing. Having been tested is a commonly cited reason for HIV test refusal [11, 31]. Dietz et al. demonstrated that patients who were tested only once in the past were more likely to refuse subsequent testing compared to patients who provided a history of multiple prior HIV tests [31]. Kalichman and Cain found that patients who were repeatedly HIV tested, as well as those who refused testing, had risk behaviors that placed them at higher risk for HIV infection compared to patients tested only once [32]. Perceived risk for HIV infection may underlie this behavior as patients who maintain a high perception of risk pursue repeat testing while those with a low perception of risk refuse subsequent testing, regardless of their actual risk. While in this study we did not specifically ask about HIV risk perception, but rather a selfperceived need for a test, we found that a low self-perceived need for testing was the strongest independent correlate for test refusal, with over a two-fold increased risk.

Perception of risk may be influenced by non-clinical factors $[33,34]$ and is frequently discordant from actual risk $[14,35,36]$. This is supported by our observation that among patients who both reported an HIV risk behavior and refused testing, the proportion who perceived a need for testing was quite low (16\%). In addition, nearly $15 \%$ of patients in our study who perceived a need for testing based on their risk of infection ultimately refused testing. In a secondary analysis restricted to participants self-reporting a perceived need for testing, we found increased refusal to be associated with either report of no HIV risk behaviors or lack of disclosure regarding HIV risk behaviors. While this difference in perception and action may be a product of competing interests such as clinical evaluation and care, it remains concerning and discrepancies in actual HIV testing rates relative to a statement of intent or plan to undergo testing have been previously described [26].

We also found that the patients enrolled in the morning were more likely to refuse testing than evening enrollees, a finding not observed by Merchant et al. in their analysis of a random sample of ED patients offered screening [13]. Other studies of routine rapid HIV testing in ED settings which may have provided information regarding patient receptiveness to testing by time of offer enrolled during limited day and evening hours and did not examine the association of test refusal with time of enrollment [10, 12, 29]. Many counselor-based programs and traditional venues for voluntary counseling and testing (VCT) operate during regular work-day hours, a schedule that may not maximize participation with routine testing. Our findings suggest that time of test offer affects the rate of refusal. The allocation of resources for ED HIV testing efforts may be optimized by focusing on higher yield times of test acceptance. 


\section{Limitations}

This study was subject to several limitations. The USHER trial was conducted at a single site, and our results may not be generalizable to other EDs. The experience of our study site may be similar, however, to those of other busy urban academic emergency departments offering routine opt-in HIV testing. The rate of test refusal in the USHER trial may be conservative as ED patients more likely to refuse HIV testing may have declined initial trial participation. However, patients enrolling in the trial were demographically similar to those who refused, with the exception of age; we did not identify age as a correlate of refusal.

Other factors that may be potentially associated with refusal of HIV testing were not assessed in this study and, as in all survey studies, it is possible that some of our measures lacked precision, potentially resulting in residual confounding despite adjustment. For example, sexual risk behaviors were limited to reports of a high risk partner, prior sexually transmitted infections, and frequency of condom use. Other sexual risk behaviors associated with HIV infection-such as heterosexual anal intercoursewere not specifically queried. Such information may have helped to inform the relationship among HIV test refusal, sexual preference and gender.

Data regarding the chief complaint or diagnoses were not collected, nor was information on length of ED visit. Racial concordance between the person offering an HIV test and the subject has previously been found to influence test acceptance [37]. In this study, however, provider/ counselor-level factors such as specific person offering the test, or racial and gender concordance could not be evaluated as the identity of the HIV counselor enrolling, counseling and testing the patient was only recorded if the participant both accepted and underwent testing. Additionally, low test offer rates in the provider arm limited our capacity to address correlates of HIV testing refusal when offered specifically by an ED provider.

This study is also susceptible to both social desirability bias and non-response bias as our survey instrument queried sensitive and potentially stigmatizing information. In an attempt to optimize data collection, we offered participants a choice between two different modes of completing a self-administered questionnaire although only $11 \%$ of respondents utilized the ACASI [38]. We also included in the analyses participants who either deliberately or inadvertently did not disclose information. We did note progressive attrition of responses to items encountered later in the questionnaire. The final 16 items referred to alcohol and illicit drug use, and we maintained a conservative definition of risk (i.e. the report of any risk behavior) in part to accommodate these missing responses.
Furthermore, in accordance with Massachusetts state law, separate written informed consent forms were required for both USHER trial enrollment and for HIV screening. The need for multiple consent processes may have limited study participation and the generalizability of our findings. Despite these limitations, the design of the USHER trial offered a unique opportunity to examine correlates of refusal. Many prior routine screening studies were unable to do so as once patients refused HIV testing no further data were collected.

\section{Conclusions}

While current CDC guidelines recommend universal screening, our findings demonstrate that routine HIV screening programs may not fully or equally engage all groups including women, patients with higher incomes and participants who did not perceive a need for HIV testing. Increased educational efforts to convey the rationale and benefits of universal screening may improve testing uptake among these groups. In addition, the modification of routine HIV screening programs to offer testing during hours of lower test refusal may increase testing rates.

Acknowledgements The authors would like to thank the co-investigators and staff of the USHER Trial, the USHER participants, and the BWH Emergency Department who made this study possible. The authors also thank Elizabeth Wright, $\mathrm{PhD}$, for critical review of the manuscript, and Amy Louise Case for technical and material support. This research was funded by the National Institute of Allergy and Infectious Diseases (T32 AI007433), National Institute of Mental Health (R01 MH073445, R01 MH65869), and the Doris Duke Charitable Foundation, Clinical Scientist Development Award to Rochelle P. Walensky.

Open Access This article is distributed under the terms of the Creative Commons Attribution Noncommercial License which permits any noncommercial use, distribution, and reproduction in any medium, provided the original author(s) and source are credited.

\section{References}

1. CDC. HIV prevalence estimates-United States, 2006. MMWR. 2008;57(29):1073-6.

2. CDC. Revised recommendations for HIV testing of adults, adolescents, and pregnant women in health-care settings. MMWR. 2006;55(RR-14):1-17.

3. Ehrenkranz PD, Ahn CJ, Metlay JP, Camargo CA Jr, Holmes WC, Rothman R. Availability of rapid human immunodeficiency virus testing in academic emergency departments. Acad Emerg Med. 2008;15(2):144-50.

4. Heyman K, Schiller J, Barnes P. Early release of selected estimates based on data from the 2007 National Health Interview Survey. National Center for Health Statistics. 2008. http:// www.cdc.gov/nchs/nhis.htm. 
5. Heron SL, Stettner E, Haley LL Jr. Racial and ethnic disparities in the emergency department: a public health perspective. Emerg Med Clin North Am. 2006;24(4):905-23.

6. Oster A, Bindman AB. Emergency department visits for ambulatory care sensitive conditions: insights into preventable hospitalizations. Med Care. 2003;41(2):198-207.

7. Blanchard JC, Haywood YC, Scott C. Racial and ethnic disparities in health: an emergency medicine perspective. Acad Emerg Med. 2003;10(11):1289-93.

8. CDC. Missed opportunities for earlier diagnosis of HIV infectionSouth Carolina, 1997-2005. MMWR. 2006;55(47):1269-72.

9. Liddicoat RV, Horton NJ, Urban R, Maier E, Christiansen D, Samet JH. Assessing missed opportunities for HIV testing in medical settings. J Gen Intern Med. 2004;19(4):349-56.

10. Brown J, Shesser R, Simon G, Bahn M, Czarnogorski M, Kuo I, et al. Routine HIV screening in the emergency department using the new US Centers for Disease Control and Prevention Guidelines: results from a high-prevalence area. J Acquir Immune Defic Syndr. 2007;46(4):395-401.

11. Liddicoat RV, Losina E, Kang M, Freedberg KA, Walensky RP. Refusing HIV testing in an urgent care setting: results from the "Think HIV" program. AIDS Patient Care STDS. 2006;20(2): 84-92.

12. Silva A, Glick NR, Lyss SB, Hutchinson AB, Gift TL, Pealer LN, et al. Implementing an HIV and sexually transmitted disease screening program in an emergency department. Ann Emerg Med. 2007;49(5):564-72.

13. Merchant RC, Seage GR, Mayer KH, Clark MA, DeGruttola VG, Becker BM. Emergency department patient acceptance of opt-in, universal, rapid HIV screening. Public Health Rep. 2008; 123(Suppl 3):27-40.

14. Groseclose SL, Erickson B, Quinn TC, Glasser D, Campbell CH, Hook EW 3rd. Characterization of patients accepting and refusing routine, voluntary HIV antibody testing in public sexually transmitted disease clinics. Sex Transm Dis. 1994;21(1):31-5.

15. Weinstock H, Dale M, Linley L, Gwinn M. Unrecognized HIV infection among patients attending sexually transmitted disease clinics. Am J Public Health. 2002;92(2):280-3.

16. Walensky RP, Arbelaez C, Reichmann WM, Walls RM, Katz JN, Block BL, et al. ) Revising expectations from rapid HIV tests in the emergency department. Ann Intern Med. 2008;149(3): 153-60.

17. Two approaches to routine HIV testing in a hospital emergency department- full text view-ClinicalTrials.gov. http://clinicaltrials. gov/ct2/show/NCT00502944?term=usher\&rank=6. Accessed 9 Oct 2009.

18. Walensky RP, Reichmann WM, Arbelaez C, Wright E, Katz JN, Seage GR, et al. Counselor- versus provider based HIV screening in the emergency department: results from the Universal screening for HIV-infection in the emergency room (USHER) randomized clinical trial. Ann Emerg Med. 2010; (supplement). In press.

19. Wuerz RC, Milne LW, Eitel DR, Travers D, Gilboy N. Reliability and validity of a new five-level triage instrument. Acad Emerg Med. 2000;7(3):236-42.

20. Hutton HE, McCaul ME, Santora PB, Erbelding EJ. The relationship between recent alcohol use and sexual behaviors: gender differences among sexually transmitted disease clinic patients. Alcohol Clin Exp Res. 2008;32(11):2008-15.

21. Raj A, Reed E, Santana MC, Walley AY, Welles SL, Horsburgh $\mathrm{CR}$, et al. The associations of binge alcohol use with HIV/STI risk and diagnosis among heterosexual African American men. Drug Alcohol Depend. 2009;101(1-2):101-6.

22. TF H-BJ Babor, Saunders JB, Monteiro MG. The alcohol use disorders identification test (AUDIT). 2nd edn. Geneva, Switzerland: World Health Organization; 2001.

23. Carey MP, Schroder KE. Development and psychometric evaluation of the brief HIV Knowledge Questionnaire. AIDS Educ Prev. 2002;14(2):172-82.

24. Zou G. A modified poisson regression approach to prospective studies with binary data. Am J Epidemiol. 2004;159(7):702-6.

25. Hutchinson AB, Corbie-Smith G, Thomas SB, Mohanan S, del Rio C. Understanding the patient's perspective on rapid and routine HIV testing in an inner-city urgent care center. AIDS Educ Prev. 2004;16(2):101-14.

26. Ostermann J, Kumar V, Pence BW, Whetten K. Trends in HIV testing and differences between planned and actual testing in the United States, 2000-2005. Arch Intern Med. 2007;167(19): 2128-35.

27. CDC. HIV/AIDS surveillance report, 2005. Vol. 17, Rev ed. Atlanta: U.S. Department of Health and Human Services, Centers for Disease Control and Prevention; 2007. p. 5-54. http:// www.cdc.gov/hiv/topics/surveillance/resources/reports/.

28. Hader SL, Smith DK, Moore JS, Holmberg SD. HIV infection in women in the United States: status at the Millennium. JAMA. 2001;285(9):1186-92.

29. Lyss SB, Branson BM, Kroc KA, Couture EF, Newman DR, Weinstein RA. Detecting unsuspected HIV infection with a rapid whole-blood HIV test in an urban emergency department. J Acquir Immune Defic Syndr. 2007;44(4):435-42.

30. Daskalakis D, Silvera R, Bernstein K, Stein D, Hagerty R, Hutt R, et al. Implementation of HIV testing at 2 New York City bathhouses: from pilot to clinical service. Clin Infect Dis. 2009;48(11):1609-16.

31. Dietz CA, Ablah E, Reznik D, Robbins DK. Patients' attitudes about rapid oral HIV screening in an urban, free dental clinic. AIDS Patient Care STDS. 2008;22(3):205-12.

32. Kalichman SC, Cain D. Repeat HIV testing and HIV transmission risk behaviors among sexually transmitted infection clinic patients. J Clin Psychol Med Settings. 2008;15(2):127-33.

33. Darrow WW, Montanea JE, Gladwin H. AIDS-related stigma among Black and Hispanic young adults. AIDS Behav. 2009;13(6):1178-88.

34. Mehrotra P, Noar SM, Zimmerman RS, Palmgreen P. Demographic and personality factors as predictors of HIV/STD partnerspecific risk perceptions: implications for interventions. AIDS Educ Prev. 2009;21(1):39-54.

35. MacKellar DA, Valleroy LA, Secura GM, Behel S, Bingham T, Celentano DD, et al. Perceptions of lifetime risk and actual risk for acquiring HIV among young men who have sex with men. AIDS Behav. 2007;11(2):263-70.

36. Boxall EH, Smith N. Antenatal screening for HIV; are those who refuse testing at higher risk than those who accept testing? J Public Health (Oxf). 2004;26(3):285-7.

37. Ford CL, Konrad TR, Godette DC, Corbie-Smith G. Acceptance of routine ELISA testing among black women STD patients: relationship to patient-provider racial concordance. Sex Transm Dis. 2008;35(3):211-3.

38. Reichmann WM, Losina E, Seage GR, Arbelaez C, Safren SA, Katz JN, et al. Does modality of survey administration impact data quality: audio computer assisted self interview (ACASI) versus self-administered pen and paper? PLoS One. 2010;5(1): e8728. 